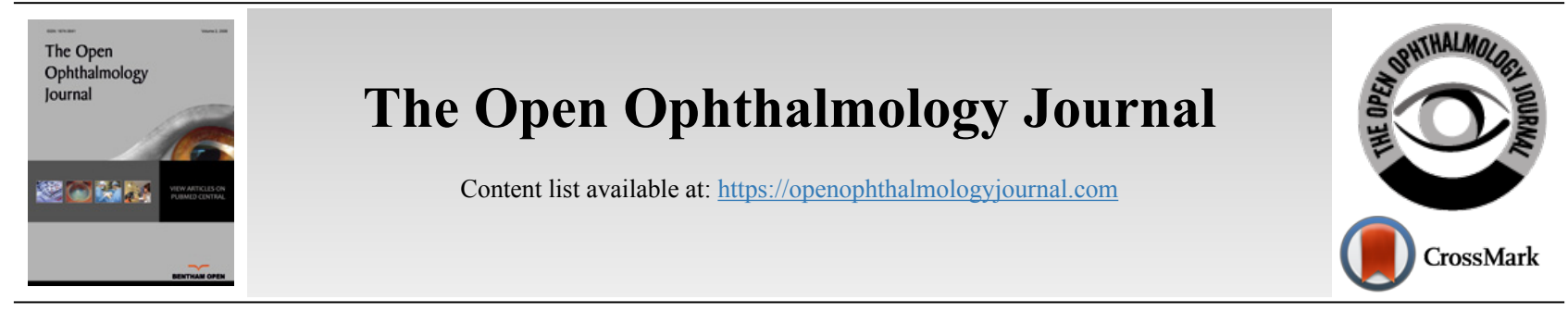

RESEARCH ARTICLE

\title{
Aqueous Flare, Functional-Morphological Parameters, and Cytokines in Age- Related Macular Degeneration after Anti-VEGF Treatment
}

\author{
Ryosuke Motohashi $^{1}$, Hidetaka Noma ${ }^{1, *}$, Kanako Yasuda ${ }^{1}$, Yuko Kasezawa $^{1}$, Hiroshi Goto ${ }^{2}$ and Masahiko Shimura ${ }^{1}$ \\ ${ }^{\prime}$ Department of Ophthalmology, Hachioji Medical Center, Tokyo Medical University, Hachioji, Tokyo 193-0998, Japan \\ ${ }^{2}$ Department of Ophthalmology, Tokyo Medical University, Tokyo, Japan
}

\begin{abstract}
:
Purpose:

The role of inflammation and cytokines in AMD and anti-Vascular Endothelial Growth Factor (anti-VEGF) treatment remains unclear. Therefore, this study aimed to examine whether anti-VEGF treatment for exudative Age-related Macular Degeneration (AMD) affects aqueous flare value (an indicator of inflammation), functional-morphologic parameters, and aqueous humor levels of cytokines or inflammatory mediators.

Methods:

We compared aqueous humor levels of 8 cytokines, growth factors (including VEGF), and inflammatory mediators in 43 patients who received anti-VEGF treatment with aflibercept for AMD and 24 healthy controls by the suspension array method. In addition, we measured aqueous flare values with a laser flare meter and Central Macular Thickness (CMT) and Macular Volume (MV) by optical coherence tomography.

Results:

The patient group had a significantly higher aqueous flare value than the control group. At baseline, CMT showed significant correlations with aqueous humor levels of soluble intercellular adhesion molecule-1 (sICAM-1), monocyte chemoattractant protein 1 (MCP-1), interleukin (IL)-6, and IL-8 and MV, with aqueous humor levels of VEGF, sICAM-1, MCP-1, IL-6, and IL-8. Moreover, we found significant correlations between aqueous flare value and aqueous humor levels of MCP-1, IL-6, IL-8, and interferon-gamma-inducible protein 10. One month after anti-VEGF treatment, the patient group showed a significant correlation between the change in MV and improvement in best-corrected visual acuity (BCVA); CMT showed no such correlation.

Conclusion:

Inflammation appears to be involved in AMD. Change in MV may be an index of improvement in BCVA in patients receiving anti-VEGF treatment for AMD.
\end{abstract}

Keywords: Age-related macular degeneration, Anti-VEGF treatment, Inflammation, Aqueous flare value, Monocyte chemoattractant protein-1, Soluble intercellular adhesion molecule-1, Interleukin, Interferon-inducible 10-kDa protein.

\begin{tabular}{|l|c|c|c|}
\hline Article History & Received: January 19, 2021 & Revised: June 14, 2021 & Accepted: June 23, 2021 \\
\hline
\end{tabular}

\section{INTRODUCTION}

About $10 \%$ of the population over the age of 50 show Agerelated Macular Degeneration (AMD), and it is the most common cause of irreversible visual loss in this age group [1]. The main feature of neovascular, i.e., exudative or "wet-type," AMD is Choroidal Neo Vascularization (CNV); this process results in sub- and intra-retinal macular edema, hemorrhage,

\footnotetext{
* Address correspondence to this author at the Department of Ophthalmology, Hachioji Medical Center, Tokyo Medical University, 1163, Tatemachi, Hachioji, Tokyo 193-0998, Japan; Tel: 81-42-665-5611; Fax: 81-42-665-1796;

E-mail: noma-hide@umin.ac.jp
}

and fibrosis, which together impair vision. CNV results from the inflammatory and angiogenic responses to damage that occurs in the outer retinal cells, Retinal Pigment Epithelium (RPE), and Bruch's membrane [2]. This damage, which can be caused by hypoxia, oxidative stress, and inflammation, induces secretion of vascular endothelial growth factor (VEGF) by cells of the RPE and growth of abnormal blood vessels from the choriocapillaris of the choroid [3, 4].

The involvement of VEGF in the pathogenesis of neovascular AMD led to the development of anti-VEGF agents. Currently, 4 such agents are used to treat AMD, 
pegaptanib, an aptamer of VEGF, bevacizumab and ranibizumab, anti-VEGF antibodies and aflibercept, a VEGF binding agent [5 - 7]. All these agents showed efficacy in improving vision in AMD in large multicenter studies [8]. However, sterile intraocular inflammation has been reported as a potential side effect of anti-VEGF treatment [9]. Of interest in this context is that inflammation is found in AMD before treatment [10]. For example, 1 study found a significantly higher aqueous flare value, an index of inflammation, in patients with AMD than in healthy controls [11]. The aqueous flare value reflects increased protein content in the anterior chamber, which results from the breakdown of the bloodocular barrier, including the Blood-Retinal Barrier (BRB), in retinal diseases and treatments [12 - 17].

In a previous study, we found significantly higher aqueous humor levels of various inflammatory mediators, including cytokines, in patients with AMD than in healthy controls [18, 19]. Despite these and other findings, the role of inflammation and cytokines in AMD and, in particular, in anti-VEGF treatment remains unclear. Therefore, we studied aqueous flare values, functional-morphologic parameters, including Central Macular Thickness (CMT) and Macular Volume (MV), and aqueous humor levels of a range of cytokines and growth factors in patients with AMD who were receiving anti-VEGF treatment. In addition, we evaluated whether these factors were correlated with each other and compared aqueous flare values with healthy controls.

\section{MATERIALS AND METHODS}

\subsection{Participants}

We performed this study at the Department of Ophthalmology, Tokyo Medical University Hachioji Medical Center, Tokyo, Japan. The study was approved by the institutional review board of Tokyo Medical University Hachioji Medical Center and conducted in accordance with the Declaration of Helsinki. All participants provided written informed consent.

The participants included 43 patients with AMD and 24 age-matched controls with healthy eyes. Patients were eligible for enrolment if they were receiving their first treatment for AMD and were treated by intravitreal aflibercept injection (IAI, Eylea, $2 \mathrm{mg}$ in $0.05 \mathrm{~mL}$, Regeneron Pharmaceuticals, Inc., Tarrytown, NY, and Bayer HealthCare Pharmaceuticals, Berlin, Germany) in the period from August 2015 to October 2018. Exclusion criteria were a history of glaucoma, uveitis, retinal disease other than $\mathrm{AMD}$, rubeosis iridis, ocular infection, laser photocoagulation, and intraocular surgery (including cataract surgery and anti-VEGF treatment). At the time of IAI, under topical anesthesia, we first collected $0.1 \mathrm{~mL}$ aqueous humor samples from the anterior chamber with a 30gauge needle attached to an insulin syringe. We immediately put the samples in sterile plastic tubes and stored them at -80 ${ }^{\circ} \mathrm{C}$ until analysis. After collecting samples, we performed IAI through the pars plana ( $3.5 \mathrm{~mm}$ from the corneal limbus). To prevent infection, we subsequently applied an antibiotic ointment to the eye once a day for 7 days.

\subsection{Evaluation of the Fundus and Clinical Parameters}

Patients were evaluated at baseline ( 1 week before IAI) and 1 month after IAI. At baseline, they showed an increased number of orange-red lesions on examination of the fundus or polypoidal lesions in indocyanine green angiography or both. On the basis of the results of the fundus examinations and indocyanine green angiographies, we diagnosed typical exudative (wet-type) AMD in 23 patients and polypoidal choroidal vasculopathy in 20 patients. No cases were found of retinal angiomatous proliferation. At the baseline visit, we also assessed Best-Corrected Visual Acuity (BCVA) as the logarithm of the minimum angle of resolution (LogMAR) and performed Optical Coherence Tomography (OCT) with a spectral-domain OCT apparatus (Spectralis, Heidelberg Engineering, Heidelberg, Germany); the built-in software in the OCT apparatus measured CMT and MV to within $6 \mathrm{~mm}$. Furthermore, we measured aqueous flare with a laser flare meter (FC-600, Kowa Co. Ltd., Tokyo, Japan), as described previously [20, 21].

One month after IAI, we completed a full eye examination (including BCVA, spectral-domain OCT, and aqueous flare), assessed vital signs, reviewed the medical history (including concomitant medications and concurrent ocular procedures) and assessed the safety of the procedure.

We evaluated the change in BCVA by calculating the difference between the values measured 1 week before and 1 month after IAI. As measures of improvement in macular edema, we calculated the percentage change in CMT and MV $(\% \mathrm{DCMT}$ and $\% \mathrm{DMV})$ as $\% \mathrm{DCMT}$ or $\% \mathrm{DMV}=\left(1-\mathrm{CMT}_{\text {post }}\right.$ or $\mathrm{MV}_{\text {post }} / \mathrm{CMT}_{\text {pre }}$ or $\left.\mathrm{MV}_{\text {pre }}\right) \times 100$, where $\mathrm{CMT}_{\text {pre }}$ and $\mathrm{MV}_{\text {pre }}$ and $\mathrm{CMT}_{\text {post }}$ and $\mathrm{MV}_{\text {post }}$ were the levels of CMT and MV 1 week before and 1 month after IAI, respectively.

Aqueous flare was measured in healthy controls at baseline and compared with the patients with AMD.

\subsection{Measurement of Cytokines and Growth Factors}

We detected the following substances in the aqueous humor samples with capture bead kits (Beadlyte, Upstate Biotechnology, Lake Placid, NY), according to the manufacturer's instructions (catalog no. HCYTOMAG-60K-08: VEGF, placental growth factor (PlGF), platelet-derived growth factor (PDGF)-AA, monocyte chemoattractant protein 1 (MCP-1), interleukin (IL)-6, IL-8, and interferon-gamma-inducible protein 10 (IP-10), catalog no. HCVD2MAG-67K-01: soluble intercellular adhesion molecule-1 (sICAM-1)). For the analysis of PlGF and sICAM1, $25 \mu \mathrm{L}$ of undiluted aqueous humor was incubated at room temperature in a dark room for 16 to 18 hours overnight; for the remaining factors, the incubation period was 2 hours. Each capture bead kit included a reference set for the concentration of each cytokine, which we used to generate duplicate standard curves. The levels in the samples were measured by suspension array technology (xMAP; Luminex Corp. Austin, TX) [18 - 22]. The levels of all the abovementioned substances were above the minimum concentration needed for detection by suspension array, VEGF: $0.64 \mathrm{pg} / \mathrm{mL}$, PIGF: $0.37 \mathrm{pg} / \mathrm{mL}$, PDGF-AA: $0.64 \mathrm{pg} / \mathrm{mL}$, sICAM-1: 0.03 
ng/mL, MCP-1: 1.2 pg/ml, IL-6: 0.29 pg/ml, IL-8: 0.14 pg/ml, and IP-10: $0.55 \mathrm{pg} / \mathrm{ml}$. To avoid analysis errors, we analyzed each patient's samples in the same run and compared them with control samples.

\subsection{Statistical Analysis}

We performed analyses with SAS 9.4 software (SAS Institute Inc., Cary, North Carolina, USA). Data are shown as means (SD) or frequencies. Continuous variables were compared between 1 week before and 1 month after IAI and between patients with AMD and healthy controls by Student's $t$ test. Correlations between variables were evaluated by Spearman's rank-order correlation analysis or Pearson's correlation analysis, as appropriate. Statistical significance was considered as $p<0.05$.

\section{RESULTS}

The AMD group consisted of 27 men and 16 women with a mean (SD) age of $73.2(10.3)$ years (Table 1), and the healthy control group consisted of 11 men and 13 women with a mean (SD) age of $75.3(4.8)$ years.

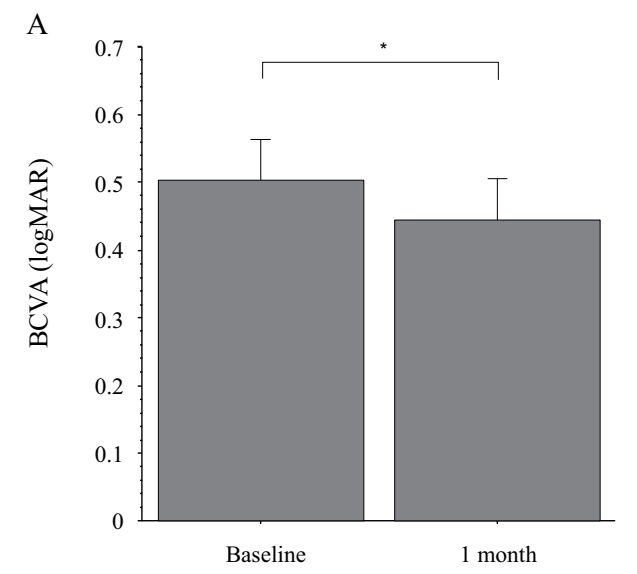

Table 1. Baseline clinical features of the AMD patients.

\begin{tabular}{|c|c|}
\hline Findings & AMD $(\mathbf{N = 4 3 )}$ \\
\hline Age (years) & $73.2 \pm 10.3^{\ddagger}$ \\
\hline Gender (female/male) & $16 / 27$ \\
\hline Baseline BCVA (logMAR) & $0.50 \pm 0.41^{\ddagger}$ \\
\hline Baseline CMT $(\mu \mathrm{m})$ & $492 \pm 227^{\ddagger}$ \\
\hline Baseline MV $\left(\mathrm{mm}^{3}\right)$ & $10.6 \pm 2.37^{\ddagger}$ \\
\hline Aqueous flare value (photon counts/ms) & $8.3 \pm 3.8^{\ddagger}$ \\
\hline
\end{tabular}

$\mathrm{AMD}=$ age-related macular degeneration; $\mathrm{BCVA}=$ best-corrected visual acuity; $\mathrm{CMT}=$ central macular thickness; $\log \mathrm{MAR}=\log$ arithm of the minimum angle of resolution; $\mathrm{MV}=$ macular volume; ${ }^{\star}$ Mean \pm standard deviation (SD)

\subsection{Examination of the Fundus and Clinical Parameters}

Mean (SD) BCVA was logMAR $0.50(0.41)$ at baseline and improved significantly to $\log$ MAR $0.44(0.40) 1$ month after IAI $(p<0.05)$ (Fig. 1A). Mean (SD) CMT was 492 (227) $\mu \mathrm{m}$ at baseline and decreased significantly to 371 (171) $\mu \mathrm{m} 1$ month after IAI $(p<0.001)$ (Fig. 1B). Mean (SD) MV was $10.6(2.37) \mathrm{mm}^{3}$ at baseline and decreased significantly to 9.3 (1.67) $\mathrm{mm}^{3} 1$ month after IAI $(p<0.001)$ (Fig. 1C).

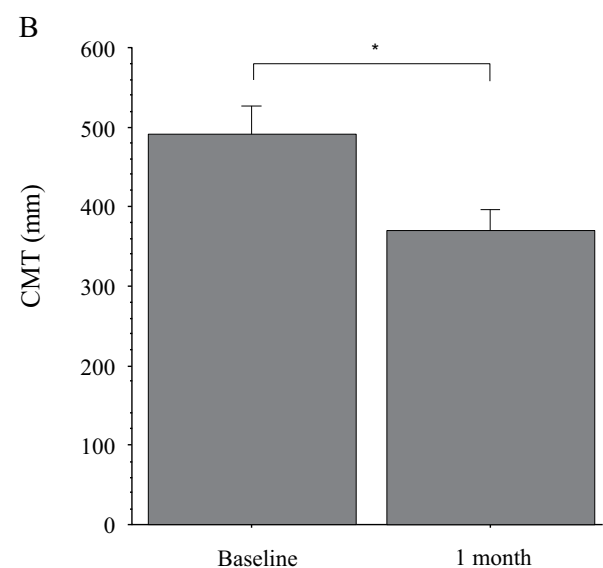

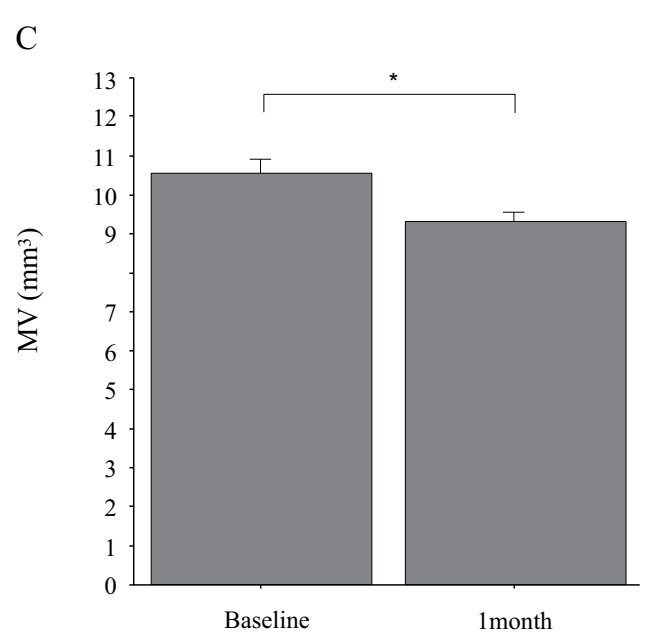

Fig. (1). Best-corrected visual acuity (BCVA), central macular thickness (CMT), and macular volume (MV) 1 month after anti-vascular endothelial growth factor (anti-VEGF) treatment by intravitreal aflibercept injection; (A) Mean (SD) baseline BCVA was logMAR 0.50 (0.41) and improved significantly to logMAR $0.44(0.40) 1$ month after anti-VEGF treatment $(P=0.041)$. (B) Mean (SD) CMT was 492 (227) $\mu$ m at baseline and decreased significantly to 371 (171) $\mu \mathrm{m} 1$ month after anti-VEGF treatment $(p<0.001)$. (C) Mean (SD) MV was $10.6(2.37) \mathrm{mm}^{3}$ at baseline and decreased significantly to $9.3(1.67) \mathrm{mm}^{3} 1$ month after anti-VEGF treatment $(p<0.001)$. 

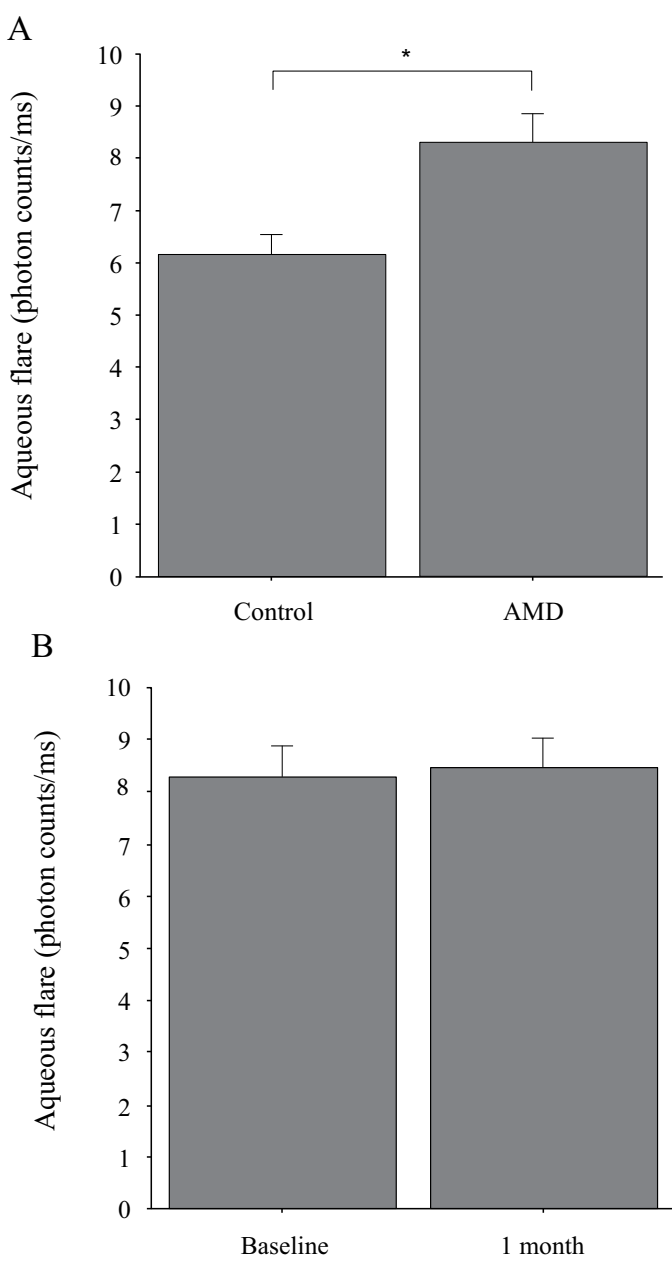

Fig. (2). The comparison of aqueous flare value in patients with age-related macular degeneration (AMD) and healthy controls and between baseline and 1 month after anti-vascular endothelial growth factor (anti-VEGF) treatment; (A) The mean (SD) aqueous flare value was significantly higher in the AMD group than in the control group (8.3 [3.8] photon counts/ms $v s .6 .1$ [2.0] photon counts/ms, respectively; $p=0.012)$. (B) The mean (SD) aqueous flare value showed no significant change from baseline to 1 month after anti-VEGF treatment (8.3 [3.8] photon counts/ms $v s$. 8.5 [3.7] photon counts/ms, respectively; $p=0.310)$.

The mean (SD) aqueous flare value was significantly higher in the AMD group than in the control group (8.3 (3.8) photon counts/ms vs. 6.1 (2.0) photon counts/ms, respectively; $p<0.05$ ) (Fig. 2A). However, it showed no significant change from baseline to 1 month after IAI (8.3 (3.8) photon counts/ms vs. 8.5 (3.7) photon counts $/ \mathrm{ms}$, respectively; $p=0.310$ ) (Fig. 2B).

\subsection{Measurement of Cytokines and Growth Factors}

At baseline, CMT correlated significantly with aqueous humor levels of sICAM-1, MCP-1, IL-6, and IL-8 but not with the levels of VEGF, PIGF, PDFG-AA, or IP-10; MV correlated significantly with the aqueous humor levels of VEGF, sICAM-1, MCP-1, IL-6, and IL-8 but not with the levels of PlGF, PDFG-AA, or IP-10; aqueous flare value correlated significantly with the aqueous humor levels of MCP-1, IL-6, IL-8, and IP-10 but not with the levels of VEGF, PIGF, PDFGAA, or sICAM-1) (Table 2).

One month after IAI, change in MV showed a significant correlation with improvement in BCVA $(\rho=0.35, P=0.022)$ but the change in CMT showed no significant correlation $(\rho=$ $0.15, P=0.329$ ) (Fig. 3A and $\mathbf{B}$ ).

Table 2. Correlations of aqueous humor levels of cytokines, growth factors, and inflammatory mediators with aqueous flare, central macular thickness, and macular volume at baseline in patients with age-related macular degeneration $(\mathrm{N}=43)$.

\begin{tabular}{|c|c|c|c|c|c|c|c|c|}
\hline \multirow{3}{*}{ Variable } & \multicolumn{9}{|c|}{ Aqueous Factors/Cytokines } \\
\cline { 2 - 10 } & VEGF & PIGF & PDGF-AA & sICAM-1 & MCP-1 & IL-6 & IL-8 & IP-10 \\
\cline { 2 - 10 } & $r$ & $r$ & $r$ & $r$ & $r$ & $r$ & $r$ & $r$ \\
$P$ value & $P$ value \\
\hline Aqueous flare & $P$ value & $P$ value & $P$ value & $P$ value & $P$ value & $P$ value & 0.45 & 0.45 \\
& -0.07 & -0.12 & -0.06 & 0.08 & 0.44 & 0.45 & 0.43 & 0.004 \\
\hline
\end{tabular}




(Table 2) contd.....
\begin{tabular}{|c|c|c|c|c|c|c|c|c|}
\hline CMT & 0.30 & -0.23 & 0.04 & 0.37 & 0.47 & 0.45 & 0.43 & 0.09 \\
& 0.071 & 0.185 & 0.823 & 0.042 & 0.007 & 0.012 & 0.016 & 0.609 \\
\hline MV & 0.47 & -0.28 & 0.12 & 0.44 & 0.51 & 0.45 & 0.42 & 0.15 \\
& 0.006 & 0.121 & 0.515 & 0.015 & 0.004 & 0.013 & 0.022 & 0.428 \\
\hline
\end{tabular}

$\mathrm{CMT}=$ central macular thickness; MV= macular volume; $\mathrm{VEGF=}$ vascular endothelial growth factor; PlGF=placental growth factor; PDGF=platelet-derived growth factor; sICAM=soluble intercellular

adhesion molecule; $\mathrm{MCP}=$ monocyte chemotactic protein; IL=interleukin; $r=$ correlation coefficient. Spearman's rank-order correlation coefficients were calculated.
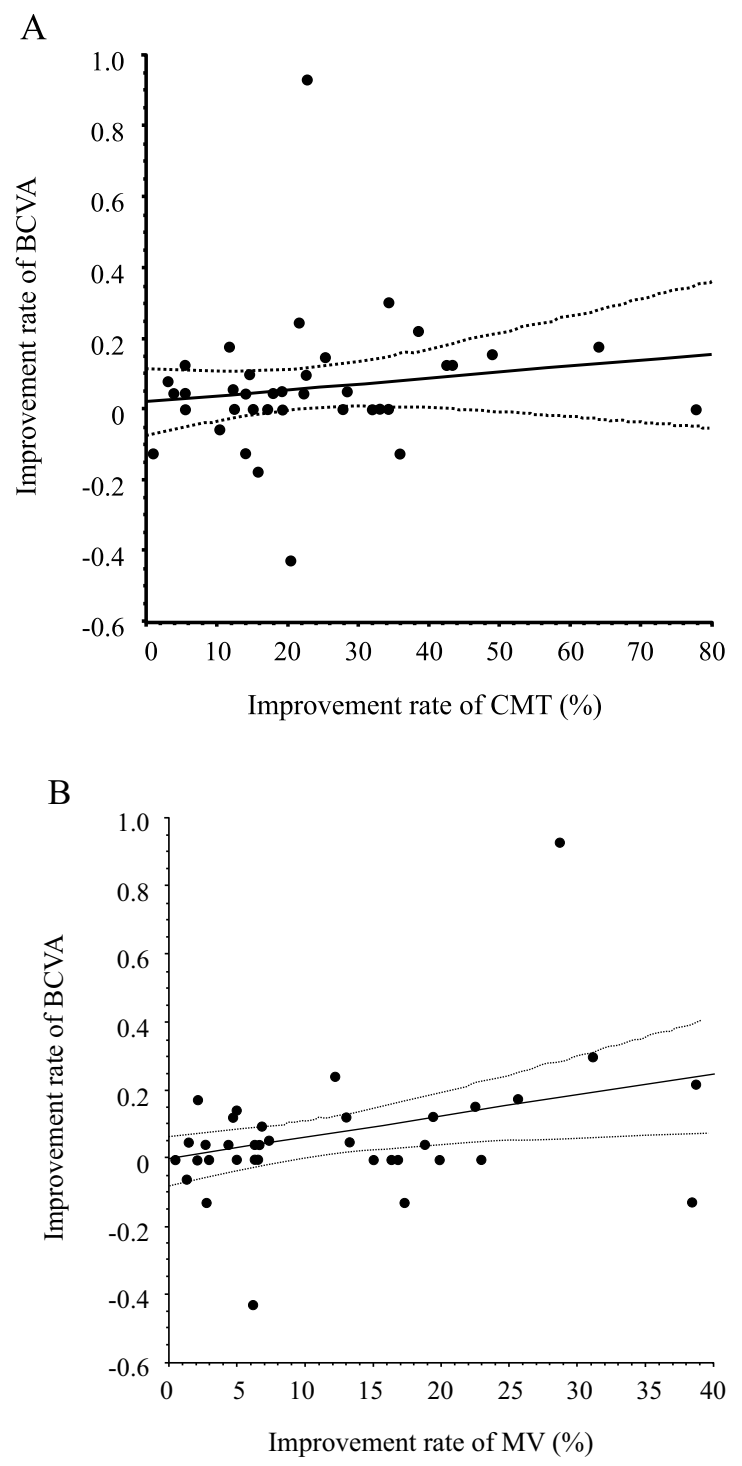

Fig. (3). Correlation between the change in central macular thickness (CMT) and macular volume (MV) and best-corrected visual acuity (BCVA) 1 month after anti-vascular endothelial growth factor (anti-VEGF) treatment; (A) The change in CMT showed no significant correlation with the improvement in BCVA 1 month after anti-VEGF treatment $(\rho=0.15, p=0.329)$. (B) The change in MV showed a significant correlation with improvement in BCVA 1 month after anti-VEGF treatment $(\rho=0.35, p=0.022)$.

\section{DISCUSSION}

The present study found that the aqueous flare value was significantly higher in the AMD group than in the control group, suggesting that inflammation is present in the eyes of patients with AMD. This finding is in line with a previous report [11] and is supported by the finding that chronic inflammation affects the pathogenesis of AMD [10]. BRB dysfunction was detected before the emergence of the clinical symptoms of AMD [23]. Classic CNV is characterized by growth through the RPE of abnormal blood vessels, which disrupts the cellular layer that forms the outer BRB. Taken together, these findings indicate that the increased permeability of neovessels in CNV impair the function of the inner BRB.

We also found evidence that higher aqueous flare values are correlated with higher aqueous humor levels of several inflammatory mediators (MCP-1, IL-6, IL-8, and IP-10). The cytokine MCP-1 recruits and activates monocytes and macrophages, and it plays a pro-inflammatory role in vascular 
remodeling by promoting infiltration of monocytes and stimulating the proliferation of smooth muscle cells [24]. Both IL-6 and IL-8 are inflammatory cytokines. IL-6 is involved in several processes, including the rearrangement of actin filaments, which can lead to the emergence of gap junctions between cells and thus increase the permeability of the endothelium [25]. IL-8, a potent chemoattractant, activates both neutrophils and $\mathrm{T}$ cells and is produced after vascular endothelial cells are exposed to hypoxia; it was found to promote angiogenesis and tumor metastasis [26]. IP-10, a CXC chemokine, is released by macrophages, endothelial cells, and fibroblasts; it is a chemoattractant for macrophages, dendritic cells, and $\mathrm{T}$ cells, is involved in type 1 immune responses mediated by $\mathrm{T}$ helper cells and generally activates cell-based immunity $[27,28]$. Thus, all 4 mediators found at higher levels in the aqueous humor in our study play a role in inflammation. As mentioned above, aqueous flare intensity is a measure of aqueous humor protein, which increases after the disruption of the blood-ocular barrier $[12,13]$. These findings and our results together indicate that in $\mathrm{AMD}$, the aqueous flare value may increase because protein leaks from iridal blood vessels after the above-mentioned inflammatory mediators disrupt the blood-ocular barrier. As a consequence, the aqueous flare value can be used to estimate the effects of these inflammatory mediators.

This study found no significant change in aqueous flare value from baseline to 1 month after IAI, which suggests that anti-VEGF treatment does not reduce inflammation in AMD. Generally, VEGF and PIGF induce leukocyte adhesion and chemotaxis and promote inflammation by binding to VEGFR-1 receptors [29 - 31], and VEGF upregulates the expression of inflammatory mediators, such as MCP-1, ICAM-1, and IL, by binding to VEGFR-2 receptors and via nuclear factor-kappa $\mathrm{B}$ [32 - 34]. In an earlier study, we found that the levels of MCP-1 and PDGF-AA in aqueous humor were significantly decreased after anti-VEGF treatment [18]. Consequently, in the current study, we expected to find that the levels of inflammatory mediators would decrease after IAI via effects at VEGFR-1 and -2 receptors; however, the results did not confirm this hypothesis.

Another of our studies found that the aqueous flare value decreased significantly after anti-VEGF treatment in both Branch Retinal Vein Occlusion (BRVO) and diabetic macular edema (DME) $[20,21]$. Therefore, we suggest that AMD does not impair the BRB as severely as BRVO or DME. Furthermore, we propose that in AMD characterized by CNV, anti-VEGF treatment might inhibit CNV more than it affects the impairment of the BRB; this hypothesis may explain why in the current study, anti-VEGF treatment did not reduce the aqueous flare value in patients with AMD. In addition, the fact that in eyes with AMD, the flare persisted after anti-VEGF treatment, which is an indication that local inflammation was still present. In fact, Sato et al. [35] reported that levels of IL-6 and IP-10 were elevated by IAI in patients with AMD, suggesting that local inflammation persists after IAI. As mentioned above, we also demonstrated that higher levels of IL-6 and IP-10 in aqueous humor play a role in inflammation. Therefore, local inflammation related to high levels of IL-6 and IP-10 may prevent a reduction of the aqueous flare value in patients with AMD.

We found a significant correlation between the change in MV but not CMT and improvement of BCVA 1 month after anti-VEGF treatment. These results suggest that the improvement of BCVA might depend on the change of MV, a measurement that reflects the entire AMD lesion. Furthermore, we found significant correlations between the aqueous humor levels of sICAM-1, MCP-1, IL-6, and IL-8 and CMT and MV and a significant correlation between the aqueous humor level of VEGF and MV. VEGF increases angiogenesis and vascular permeability in the retina by changing the arrangement of tight junctions in vascular endothelial cells [36 - 38]. Researchers suggested that VEGF-related disruption of the BRB depends partly on leukocytes because treatment of rat eyes with VEGF inhibited the expression of ICAM-1, which is expressed on leukocytes and promotes their adhesion and migration and prevents the breakdown of the BRB [39]. The same study found that blocking ICAM-1 with a neutralizing antibody suppressed retinal leukostasis [39]. Taken together, these findings indicate that VEGF increases the expression of ICAM-1 in vitro in capillary endothelial cells and in vivo in the retinal vasculature. Thus, increased VEGF production may increase retinal expression of ICAM-1 and thus mediate leukocyte adhesion to retinal blood vessels. This hypothesis is in line with the above-mentioned findings that VEGF induces MCP-1, IL-6, and IL-8 via VEGFR-1 and -2 receptors [2934]. Furthermore, it explains how the suppression of VEGF activity with an anti-VEGF agent decreases angiogenesis and vascular permeability in a large area of the macula and why we found a significant correlation between the improvement in BCVA and MV 1 month after IAI. When taken together with the reports discussed above, our findings suggest that MV may reflect AMD lesions and that a change in MV may be useful as an index of improvement in BCVA in patients with AMD receiving treatment with an anti-VEGF agent.

The present study has several limitations. First, the number of patients with AMD was relatively small. Second, aqueous humor cytokines were evaluated only before IAI and not measured afterwards. Future studies should measure cytokines also after IAI to further elucidate the pathogenesis of AMD by examining the relationship between changes of cytokine levels and both changes in the aqueous flare value and improvement in clinical findings after IAI in patients with AMD.

\section{CONCLUSION}

In summary, in our study, the aqueous flare value was significantly higher in the AMD group than in the control group. At baseline, we found significant correlations between the CMT and the aqueous humor levels of SICAM-1, MCP-1, IL-6, and IL-8 and between the MV and the aqueous humor levels of VEGF, sICAM-1, MCP-1, IL-6, and IL-8. Moreover, we found significant correlations between the aqueous flare value and the aqueous humor levels of MCP-1, IL-6, IL-8, and IP-10. The change in MV but not CMT showed a significant correlation with an improvement in BCVA 1 month after antiVEGF treatment by IAI. These findings suggest that inflammation is involved in the pathogenesis of AMD and that the change in MV may represent an index of the improvement 
of BCVA in patients with AMD receiving anti-VEGF treatment.

\section{AUTHORS' CONTRIBUTIONS}

H.N., and M.S. were involved in the design and conduction of the study. The collection and management of the data were done by R.M., K.Y., H.N., and M.S., while analysis and interpretation of the data were performed by K.Y., and M.S.. The preparation of the first draft of the manuscript was done by H.N., and the review was performed and the approval of the manuscript was given by H.G., and M.S. All authors have read and approved the final manuscript.

\section{ETHICS APPROVAL AND CONSENT TO PARTICIPATE}

This study was approved by the Institutional Review Board of Tokyo Medical University Hachioji Medical Center, Hachioji, Tokyo, Japan (H-107).

\section{HUMAN AND ANIMAL RIGHTS}

No animals were used in this research. All human research procedures were followed in accordance with the ethical standards of the committee responsible for human experimentation (institutional and national), and with the Helsinki Declaration of 1975, as revised in 2013.

\section{CONSENT FOR PUBLICATION}

All participants provided written informed consent.

\section{AVAILABILITY OF DATA AND MATERIALS}

Not applicable.

\section{FUNDING}

None.

\section{CONFLICT OF INTEREST}

The authors declare no conflict of interest, financial or otherwise.

\section{ACKNOWLEDGEMENTS}

We thank Katsunori Shimada (Department of Biostatistics, STATZ Corporation, Tokyo) for his assistance in the statistical analysis.

\section{REFERENCES}

[1] Telander DG. Inflammation and age-related macular degeneration (AMD). Semin Ophthalmol 2011; 26(3): 192-7.

[http://dx.doi.org/10.3109/08820538.2011.570849] [PMID: 21609232]

[2] Jager RD, Mieler WF, Miller JW. Age-related macular degeneration. N Engl J Med 2008; 358(24): 2606-17. [http://dx.doi.org/10.1056/NEJMra0801537] [PMID: 18550876]

[3] Rosenfeld PJ, Brown DM, Heier JS, et al. Ranibizumab for neovascular age-related macular degeneration. N Engl J Med 2006; 355(14): 1419-31.

[http://dx.doi.org/10.1056/NEJMoa054481] [PMID: 17021318]

[4] Kokotas H, Grigoriadou M, Petersen MB. Age-related macular degeneration: genetic and clinical findings. Clin Chem Lab Med 2011; 49(4): 601-16.

[http://dx.doi.org/10.1515/CCLM.2011.091] [PMID: 21175380]

[5] Carmeliet P, Moons L, Luttun A, et al. Synergism between vascular endothelial growth factor and placental growth factor contributes to angiogenesis and plasma extravasation in pathological conditions. Nat Med 2001; 7(5): 575-83.

[http://dx.doi.org/10.1038/87904] [PMID: 11329059]

[6] Holash J, Davis S, Papadopoulos N, et al. VEGF-Trap: a VEGF blocker with potent antitumor effects. Proc Natl Acad Sci USA 2002; 99(17): 11393-8.

[http://dx.doi.org/10.1073/pnas.172398299] [PMID: 12177445]

[7] Heier JS, Antoszyk AN, Pavan PR, et al. Ranibizumab for treatment of neovascular age-related macular degeneration: a phase I/II multicenter, controlled, multidose study. Ophthalmology 2006; 113(4): e631-634.

[8] Heier JS, Brown DM, Chong V, et al. Intravitreal aflibercept (VEGF trap-eye) in wet age-related macular degeneration. Ophthalmology 2012; 119(12): 2537-48.

[http://dx.doi.org/10.1016/j.ophtha.2012.09.006] [PMID: 23084240]

[9] Agrawal S, Joshi M, Christoforidis JB. Vitreous inflammation associated with intravitreal anti-VEGF pharmacotherapy. Mediators Inflamm 2013; 2013(10): 943409.

[http://dx.doi.org/10.1155/2013/943409] [PMID: 24307762]

[10] Kauppinen A, Paterno JJ, Blasiak J, Salminen A, Kaarniranta K. Inflammation and its role in age-related macular degeneration. Cell Mol Life Sci 2016; 73(9): 1765-86.

[http://dx.doi.org/10.1007/s00018-016-2147-8] [PMID: 26852158]

[11] Kubota T, Küchle M, Nguyen NX. Aqueous flare in eyes with agerelated macular degeneration. Jpn J Ophthalmol 1994; 38(1): 67-70. [PMID: 7933700]

[12] Ladas JG, Wheeler NC, Morhun PJ, Rimmer SO, Holland GN. Laser flare-cell photometry: methodology and clinical applications. Surv Ophthalmol 2005; 50(1): 27-47.

[http://dx.doi.org/10.1016/j.survophthal.2004.10.004] [PMID: 15621076]

[13] Tugal-Tutkun I, Herbort CP. Laser flare photometry: a noninvasive, objective, and quantitative method to measure intraocular inflammation. Int Ophthalmol 2010; 30(5): 453-64.

[http://dx.doi.org/10.1007/s10792-009-9310-2] [PMID: 19430730]

[14] Miyake K, Miyake T, Kayazawa F. Blood-aqueous barrier in eyes with retinal vein occlusion. Ophthalmology 1992; 99(6): 906-10.

[http://dx.doi.org/10.1016/S0161-6420(92)31875-5] [PMID: 1630780]

[15] Nguyen NX, Küchle M. Aqueous flare and cells in eyes with retinal vein occlusion--correlation with retinal fluorescein angiographic findings. Br J Ophthalmol 1993; 77(5): 280-3.

[http://dx.doi.org/10.1136/bjo.77.5.280] [PMID: 8318463]

[16] Kubota T, Motomatsu K, Sakamoto M, Honda T, Ishibashi T. Aqueous flare in eyes with senile disciform macular degeneration: correlation with clinical stage and area of neovascular membrane. Graefes Arch Clin Exp Ophthalmol 1996; 234(5): 285-7.

[http://dx.doi.org/10.1007/BF00220701] [PMID: 8740247]

[17] Zaczek A, Hallnäs K, Zetterström C. Aqueous flare intensity in relation to different stages of diabetic retinopathy. Eur J Ophthalmol 1999; 9(3): 158-64.

[http://dx.doi.org/10.1177/112067219900900303] [PMID: 10544971]

[18] Motohashi R, Noma H, Yasuda K, Kotake O, Goto H, Shimura M. Dynamics of inflammatory factors in aqueous humor during ranibizumab or aflibercept treatment for age-related macular degeneration. Ophthalmic Res 2017; 58(4): 209-16.

[http://dx.doi.org/10.1159/000478705] [PMID: 28796997]

[19] Motohashi R, Noma H, Yasuda K, Kotake O, Goto H, Shimura M. Dynamics of soluble vascular endothelial growth factor receptors and their ligands in aqueous humour during ranibizumab for age-related macular degeneration. J Inflamm (Lond) 2018; 15: 26.

[http://dx.doi.org/10.1186/s12950-018-0203-x] [PMID: 30534004]

[20] Noma H, Mimura T, Yasuda K, Shimura M. Functional-morphological parameters, aqueous flare and cytokines in macular oedema with branch retinal vein occlusion after ranibizumab. Br J Ophthalmol 2017; 101(2): 180-5.

[http://dx.doi.org/10.1136/bjophthalmol-2015-307989]

[PMID: 27073207]

[21] Imazeki M, Noma H, Yasuda K, Motohashi R, Goto H, Shimura M. Anti-VEGF therapy reduces inflammation in diabetic macular edema. Ophthalmic Res 2021; 64(1): 43-9. [PMID: 32454504]

[22] Noma H, Mimura T, Yasuda K, Motohashi R, Kotake O, Shimura M. Aqueous humor levels of soluble vascular endothelial growth factor receptor and inflammatory factors in diabetic macular edema. Ophthalmologica 2017; 238(1-2): 81-8.

[http://dx.doi.org/10.1159/000475603] [PMID: 28564655]

[23] Silva R, Cachulo ML, Fonseca P, et al. Age-related macular 
degeneration and risk factors for the development of choroidal neovascularisation in the fellow eye: a 3-year follow-up study. Ophthalmologica 2011; 226(3): 110-8.

[http://dx.doi.org/10.1159/000329473] [PMID: 21822000]

[24] Schober A, Zernecke A. Chemokines in vascular remodeling. Thromb Haemost 2007; 97(5): 730-7.

[http://dx.doi.org/10.1160/TH07-02-0085] [PMID: 17479183]

[25] Maruo N, Morita I, Shirao M, Murota S. IL-6 increases endothelial permeability in vitro. Endocrinology 1992; 131(2): 710-4. [PMID: 1639018]

[26] Taub DD, Anver M, Oppenheim JJ, Longo DL, Murphy WJ. T lymphocyte recruitment by interleukin-8 (IL-8). IL-8-induced degranulation of neutrophils releases potent chemoattractants for human T lymphocytes both in vitro and in vivo. J Clin Invest 1996; 97(8): 1931-41

[http://dx.doi.org/10.1172/JCI118625] [PMID: 8621778]

[27] Feldman ED, Weinreich DM, Carroll NM, et al. Interferon gammainducible protein 10 selectively inhibits proliferation and induces apoptosis in endothelial cells. Ann Surg Oncol 2006; 13(1): 125-33. [http://dx.doi.org/10.1245/ASO.2006.03.038] [PMID: 16378159]

[28] Bodnar RJ, Yates CC, Wells A. IP-10 blocks vascular endothelial growth factor-induced endothelial cell motility and tube formation via inhibition of calpain. Circ Res 2006; 98(5): 617-25.

[http://dx.doi.org/10.1161/01.RES.0000209968.66606.10] [PMID: 16484616]

[29] Clauss M, Weich H, Breier G, et al. The vascular endothelial growth factor receptor Flt-1 mediates biological activities. Implications for a functional role of placenta growth factor in monocyte activation and chemotaxis. J Biol Chem 1996; 271(30): 17629-34.

[http://dx.doi.org/10.1074/jbc.271.30.17629] [PMID: 8663424]

[30] Luttun A, Tjwa M, Moons L, et al. Revascularization of ischemic tissues by PlGF treatment, and inhibition of tumor angiogenesis, arthritis and atherosclerosis by anti-Flt1. Nat Med 2002; 8(8): 831-40. [http://dx.doi.org/10.1038/nm731] [PMID: 12091877]

[31] Shibuya M. Vascular Endothelial Growth Factor (VEGF) and Its Receptor (VEGFR) Signaling in Angiogenesis: A Crucial Target for Anti- and Pro-Angiogenic Therapies. Genes Cancer 2011; 2(12): 1097-105.

[http://dx.doi.org/10.1177/1947601911423031] [PMID: 22866201]
[32] Ledebur HC, Parks TP. Transcriptional regulation of the intercellular adhesion molecule-1 gene by inflammatory cytokines in human endothelial cells. Essential roles of a variant NF-kappa B site and p65 homodimers. J Biol Chem 1995; 270(2): 933-43. [http://dx.doi.org/10.1074/jbc.270.2.933] [PMID: 7822333]

[33] Baldwin AS Jr. The NF-kappa B and I kappa B proteins: new discoveries and insights. Annu Rev Immunol 1996; 14: 649-83. [http://dx.doi.org/10.1146/annurev.immunol.14.1.649] [PMID: 8717528]

[34] Marumo T, Schini-Kerth VB, Fisslthaler B, Busse R. Platelet-derived growth factor-stimulated superoxide anion production modulates activation of transcription factor NF-kappaB and expression of monocyte chemoattractant protein 1 in human aortic smooth muscle cells. Circulation 1997; 96(7): 2361-7.

[http://dx.doi.org/10.1161/01.CIR.96.7.2361] [PMID: 9337212]

[35] Sato T, Takeuchi M, Karasawa Y, Enoki T, Ito M. Intraocular inflammatory cytokines in patients with neovascular age-related macular degeneration before and after initiation of intravitreal injection of anti-VEGF inhibitor. Sci Rep 2018; 8(1): 1098. [http://dx.doi.org/10.1038/s41598-018-19594-6] [PMID: 29348424]

[36] Dvorak HF, Brown LF, Detmar M, Dvorak AM. Vascular permeability factor/vascular endothelial growth factor, microvascular hyperpermeability, and angiogenesis. Am J Pathol 1995; 146(5): 1029-39. [PMID: 7538264]

[37] Vinores SA, Derevjanik NL, Ozaki H, Okamoto N, Campochiaro PA Cellular mechanisms of blood-retinal barrier dysfunction in macular edema. Doc Ophthalmol 1999; 97(3-4): 217-28.

[http://dx.doi.org/10.1023/A:1002136712070] [PMID: 10896335]

[38] Gardner TW, Antonetti DA, Barber AJ, Lieth E, Tarbell JA. The molecular structure and function of the inner blood-retinal barrier. Doc Ophthalmol 1999; 97(3-4): 229-37.

[http://dx.doi.org/10.1023/A:1002140812979] [PMID: 10896336]

[39] Miyamoto K, Khosrof S, Bursell SE, et al. Prevention of leukostasis and vascular leakage in streptozotocin-induced diabetic retinopathy via intercellular adhesion molecule-1 inhibition. Proc Natl Acad Sci USA 1999; 96(19): 10836-41.

[http://dx.doi.org/10.1073/pnas.96.19.10836] [PMID: 10485912]

\section{(C) 2021 Motohashi et al.}

This is an open access article distributed under the terms of the Creative Commons Attribution 4.0 International Public License (CC-BY 4.0), a copy of which is available at: https://creativecommons.org/licenses/by/4.0/legalcode. This license permits unrestricted use, distribution, and reproduction in any medium, provided the original author and source are credited. 